\title{
Children hospitalized for juvenile arthritis in the United States
}

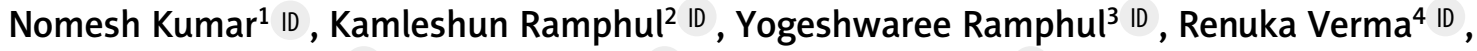 \\ Stephanie G. Mejias ${ }^{5}$ ID , Petras Lohana $^{1}$ ID , Jyotsnav Joynauth $^{6} \mathrm{ID}$ \\ ${ }^{1}$ Department of Medicine, Liaquat University of Medical and Health Sciences Hospital, Karachi, Pakistan \\ 2Department of Pediatrics, Shanghai Xin Hua Hospital, Shanghai Jiao Tong University, School of Medicine, Shanghai, China \\ ${ }^{3}$ Department of Medicine, Sir Seewoosagur Ramgoolam National Hospital, Pamplemousses, Mauritius \\ ${ }^{4}$ Department of Internal Medicine, Guru Gobind Singh Medical College, Punjab, India \\ ${ }^{5}$ Department of Pediatrics, The University 'beroamericana Unibe School of Medicine/Robert Reid Cabral Children's Hospital, \\ Santo Domingo, Dominicana \\ ${ }^{6}$ Department of Pediatrics, the Children's Hospital, Zhejiang University School of Medicine, Hangzhou, Zhejiang Province, China
}

\begin{abstract}
Juvenile arthritis (JA) is an autoimmune condition affecting children. We used the 2017 National Inpatient Sample (NIS) to evaluate the impact of JA in the United States. The admission data were converted to weighted form and patients between the ages of 0 and 18 (inclusive) were used in our study. 560 weighted cases were found in 2017. It was more common in females than males (340 females, 220 males), Whites (235 cases), and Medicaid covered $61.6 \%$ of all patients with JA (345 cases). The total hospital charges were $\$ 25,147,389$ while the mean length of stay (LOS) was 4.55 days. The highest number of cases was reported in April 2017.
\end{abstract}

Key words: children, juvenile arthritis, Healthcare Cost and Utilization Project.

\section{Introduction}

Juvenile arthritis (JA) usually refers to autoimmune inflammation of joints in children. The most common subtype of JA is juvenile idiopathic arthritis (JIA). Juvenile arthritis is believed to affect children of all ages and races. It presents with swelling, stiffness, and pain of joints. It can be worse in the morning. Depending on the subtypes, other clinical findings can also involve high fever, rash, and lymphadenopathy [1].

Since there are limited data available on the impact of JA in the United States, our study aims to provide an epidemiological perspective on the incidence of JA and its burden, as well as a comparison with available data from other countries.

\section{Material and methods Study design}

We used de-identified data from the 2017 National Inpatient Sample (NIS) provided by the Healthcare Cost and Utilization Project (HCUP), Agency for Healthcare
Research and Quality, and their multiple partners, to conduct a retrospective study. The 2017 NIS is one of the biggest nationally representative databases available for patients in the United States. It contains diagnosis, hospital, and procedural records, as well as several characteristics of each patient including their age, gender, race, and insurance form [2].

\section{Identification of sample}

All patients of ages 18 and below were retained for our analysis. International Classification of Diseases, $10^{\text {th }}$ Revision, Clinical Modification (ICD-10-CM) codes for juvenile arthritis, provided by HCUP, was applied to identify patients hospitalized with a primary diagnosis [2].

\section{Statistical analysis}

Following the guidelines set by the provider, the data were converted to "weighted form" for a national estimate [2]. The total number of cases and patient variables such as age, gender, race, insurance form, as well as distribution in the month of admission were studied.

Address for correspondence:

Kamleshun Ramphul, Department of Pediatrics, Shanghai Xin Hua Hospital, Shanghai Jiao Tong University, School of Medicine, 1665 Kongjiang Rd, Yangpu District, Shanghai, 200092, China, e-mail: adramphul@hotmail.com Submitted: 22.03.2021; Accepted: 27.07.2021 
Differences among categorical variables were compared as appropriate using Pearson's $\chi^{2}$ tests. All results with a $p$-value less than 0.05 were considered statistically significant.

We also calculated their mean length of stay (LOS) and hospital charges, and used the Kaiser Family Foundation (KFF) data to estimate the number of patients with JA admitted per 100,000 children in the United States in 2017 [3]. Our statistical analysis was executed via SPSS Statistics, version 26 (IBM Corporation, Armonk, NY).

\section{Results}

Between January 1 1st 2017 and December 31 $1^{\text {st }}$, 2017, 560 weighted cases with a primary discharge code of juvenile arthritis were recorded among children of ages 18 and less in the United States, with a national incidence of 7.4 cases per 100,000 children.

The condition was more common in females, with 340 cases (60.7\%, 12.5 admissions for JA per 100,000 hospitalizations among females), while 220 cases were found among males (39.3\%, 7.9 admissions for JA per 100,000 hospitalizations among males, $p<0.01$ ).

Although a higher number of patients were classified as Caucasian (235 cases, 44.8\%), the incidence among admissions was higher among Hispanics (12.4 cases of JA per 100,000 admissions). Medicaid was the most common insurance, which provided for 345 cases (61.6\%) (Table I).

Our study also found that the mean age of patients hospitalized with a primary diagnosis of JA was 9.22 years (IQR: $5.00-13.75$ years). Their mean LOS was 4.55 days (IQR: 2.00-6.00 days).

The mean and total hospital charges were $\$ 44,906.05$ (IQR: \$16,591.28-\$57,336.83) and \$25,147,389 respectively. Our study failed to show any statistically significant distribution in terms of ages between the children. The highest number of cases was recorded during April in 2017 (Fig. 1) and spring (33.9\% in spring, 25\% in summer, $25 \%$ in winter, and $16.1 \%$ in autumn).

\section{Discussion}

Our investigation gives a fresh understanding of the prevalence of JA among hospitalized children and its economic burden. The annual incidence rate of JA in the United States (7.4 cases per 100,000 children) was lower than several Nordic countries and Sweden (15 cases per 100,000 children and 12.8 cases per 100,000 children) [4], Canada (17.8 cases per 100,000 children) [5], and India (48 cases per 100,000 children) [6], but higher than France (3.1 cases per 100,000 children) [7], Taiwan (4.93 cases per 100,000 children) [8], Singapore (5.8 cases per 100,000 children) [9], and Spain (6.9 cases per 100,000 children)
Table I. Characteristics of patients aged 0-18 admitted for juvenile arthritis in the United States

\begin{tabular}{|c|c|c|c|}
\hline Parameters & $\begin{array}{c}\text { Number } \\
\text { of cases } \\
(\%)\end{array}$ & $\begin{array}{c}\text { Number of cases } \\
\text { per } 100,000 \\
\text { admissions }\end{array}$ & $p$-value \\
\hline \multicolumn{3}{|l|}{ Gender } & \multirow[t]{3}{*}{$<0.01$} \\
\hline Male & $220(39.3)$ & 7.9 & \\
\hline Female & $340(60.7)$ & 12.5 & \\
\hline \multicolumn{3}{|l|}{ Race } & \multirow[t]{4}{*}{$<0.01$} \\
\hline Caucasian & $235(44.8)$ & 9.22 & \\
\hline African American & 75 (14.3) & 9.25 & \\
\hline Hispanic & $130(24.8)$ & 12.4 & \\
\hline \multicolumn{3}{|l|}{$\begin{array}{l}\text { Primary expected } \\
\text { payer form }\end{array}$} & \multirow[t]{3}{*}{$<0.01$} \\
\hline Medicaid & $345(61.6)$ & 12.7 & \\
\hline Private & $170(30.4)$ & 7.1 & \\
\hline
\end{tabular}

[10]. The incidence of JA in the United States in previous studies varied between 4.1 and 11.9 cases per 100,000 based on geographical areas and study designs [11-13].

Berthold et al. [4] proposed that there are several genetic as well as external factors (climatic and infectious) that can predispose children to a higher risk of JA. They also found that the incidence varied throughout Europe, with a "north-south gradient", where the cases were higher in the north and started to decrease in more southerly European populations.

Our study does not allow us to accurately compare the incidence of JA in different states and counties of the United States, and we strongly encourage future studies to consider including it in their analysis.

Beukelman et al. [14] compiled data from several international registries and studies. We believe that a similar analysis through different states and counties

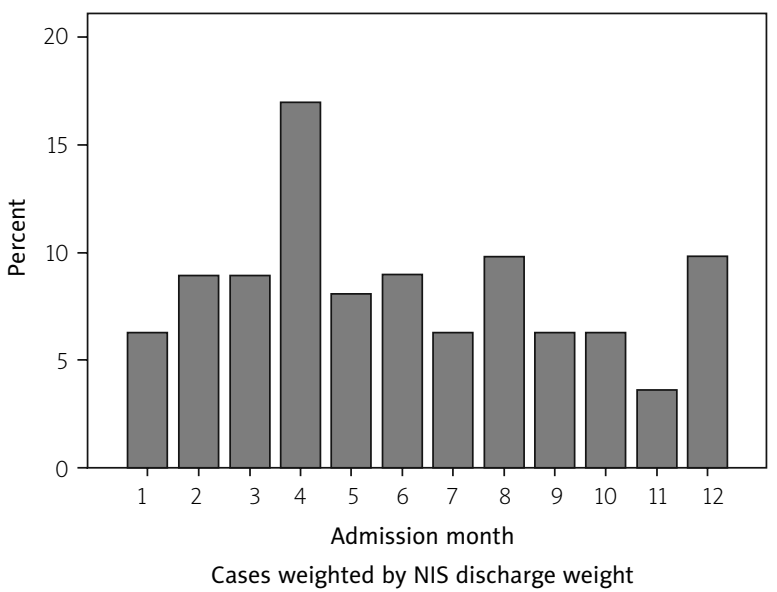

Fig. 1. Cases weighted by National Inpatient Sample (NIS) discharge weight. 
in the United States can help understand geographical differences better.

A higher incidence was also found among females compared to males in the United States, as well as a higher ethnic risk among Hispanics in our study. While the difference in risks and prevalence between males and females for autoimmune conditions is less conspicuous among children as compared to adults, several studies have shown a strong predisposition for some autoimmune conditions such as JA among female children [15].

Ringold et al. [16] reported a higher number of Caucasian children having JIA in their study, and Pelajo et al. [17] associated Hispanics with a more complicated outcome and possible disabilities.

The highest number of cases was reported in April (Spring) in our report, correlating with some studies that have linked their incidence with a seasonal pattern [18]. However, we believe a bigger study sample over several years might provide a stronger conclusion for the United States.

The main shortcoming of our study is the inability to follow up the patients via the HCUP database and determine the incidence per subtype of JA.

However, the analysis provides a window of opportunity for pediatric rheumatologists to further explore the different missing pieces in the puzzle to fully understand the condition.

\section{Conclusions}

The present study found that the incidence rate of JA was 7.4 cases per 100,000 children, with a higher risk in females and Hispanics. The incidence rate of JA in the US is less than that reported in the Scandinavian countries, and is comparable to other European countries.

\section{Acknowledgements}

The authors would like to express its sincere gratitude to HCUP, the Agency for Healthcare Research and Quality, and their multiple partners, for granting us access to the database.

Additional information can be found at: https://www. hcup-us.ahrq.gov/team/partners/partners_list.jsp.

\section{The authors declare no conflict of interest.}

\section{References}

1. National Institute of Arthritis and Musculoskeletal and Skin Diseases (NIH). Juvenile arthritis [cited $202122^{\text {nd }}$ March]; Available from: https://www.niams.nih.gov/health-topics/ juvenile-arthritis\#tab-overview.

2. Healthcare Cost and Utilization Project (HCUP). HCUP National Inpatient Sample (NIS). 2017. Agency for Healthcare Re- search and Quality, Rockville, MD [cited 2020 July 1st]; Available from: https://www.hcup-us.ahrq.gov/nisoverview.jsp.

3. State Health Facts, Population Distribution by Age, KFF, 2021 [cited May, 1, 2021]. Available from: https://www.kff.org/other/ state-indicator/distribution-by-age/

4. Berthold E, Månsson B, Kahn R. Outcome in juvenile idiopathic arthritis: a population-based study from Sweden. Arthritis Res Ther 2019; 21: 218, DOI: 10.1186/s13075-019-1994-8.

5. Ehrmann Feldman D, Bernatsky S, Houde M. The incidence of juvenile rheumatoid arthritis in Quebec: a population databased study. Pediatr Rheumatol Online J 2009; 7: 20, DOI: 10.1186/1546-0096-7-20.

6. Abujam B, Mishra R, Aggarwal A. Prevalence of musculoskeletal complaints and juvenile idiopathic arthritis in children from a developing country: a school-based study. Int J Rheum Dis 2014; 17: 256-260, DOI: 10.1111/1756-185X.12276.

7. Danner S, Sordet C, Terzic J, et al. Epidemiology of juvenile idiopathic arthritis in Alsace, France. J Rheumatol 2006; 33: 1377-1381.

8. $\mathrm{Yu} \mathrm{HH}$, Chen PC, Wang LC, et al. Juvenile idiopathic arthritis-associated uveitis: a nationwide population-based study in Taiwan. PLoS One 2013; 8: e70625, DOI: 10.1371/journal. pone.0070625.

9. Ng PC, Ooi PL, Ang E, et al. SAT0476 juvenile idiopathic arthritis in Singapore: a 10-year clinical experience. Ann Rheum Dis 2019; 78 (Suppl 2): 1326-1326, DOI: 10.1136/annrheumdis-2019-eular.3593.

10. Modesto C, Antón J, Rodriguez B, et al. Incidence and prevalence of juvenile idiopathic arthritis in Catalonia (Spain). Scand J Rheumatol 2010; 39: 472-479, DOI: 10.3109/03009741003742722.

11. Macaubas C, Nguyen K, Milojevic D, et al. Oligoarticular and polyarticular JIA: epidemiology and pathogenesis. Nat Rev Rheumatol 2009; 5: 616-626, DOI: 10.1038/nrrheum.2009.209.

12. Harrold LR, Salman C, Shoor S, et al. Incidence and prevalence of juvenile idiopathic arthritis among children in a managed care population, 1996-2009. J Rheumatol 2013; 40: 12181225, DOI: 10.3899/jrheum.120661.

13. Krause ML, Crowson CS, Michet CJ, et al. Juvenile idiopathic arthritis in Olmsted County, Minnesota, 1960-2013. Arthritis Rheumatol 2016; 68: 247-254, DOI: 10.1002/art.39323.

14. Beukelman T, Anink J, Berntson L, et al. A survey of national and multi-national registries and cohort studies in juvenile idiopathic arthritis: challenges and opportunities. Pediatr Rheumatol Online J 2017; 15: 31, DOI: 10.1186/s12969-017-0161-5.

15. Cattalini M, Soliani M, Caparello MC, Cimaz R. Sex differences in pediatric rheumatology. Clin Rev Allergy Immunol 2019; 56: 293-307, DOI: 10.1007/s12016-017-8642-3.

16. Ringold S, Beukelman T, Nigrovic PA, et al. Race, ethnicity, and disease outcomes in juvenile idiopathic arthritis: a cross-sectional analysis of the Childhood Arthritis and Rheumatology Research Alliance (CARRA) Registry. J Rheumatol 2013; 40: 936-942, DOI: 10.3899/jrheum.121147.

17. Pelajo CF, Angeles-Han ST, Prahalad S, et al. Evaluation of the association between Hispanic ethnicity and disease activity and severity in a large cohort of patients with juvenile idiopathic arthritis. Rheumatol Int 2013; 33: 2549-2554, DOI: 10.1007/s00296-013-2773-5.

18. Feldman BM, Birdi N, Boone JE, et al. Seasonal onset of systemic-onset juvenile rheumatoid arthritis. J Pediatr 1996; 129: 513-518, DOI: 10.1016/s0022-3476(96)70115-4. 